\title{
Wide-pressure-range SEMs and modelling
}

XVP FOR EVO SEMS

\section{LEO}

\author{
www.leo-usa.com
}

Extended variable pressure (XVP) technology

LEO Electron Microscopy Group have introduced XVP technology as an integral part of their new EVO family

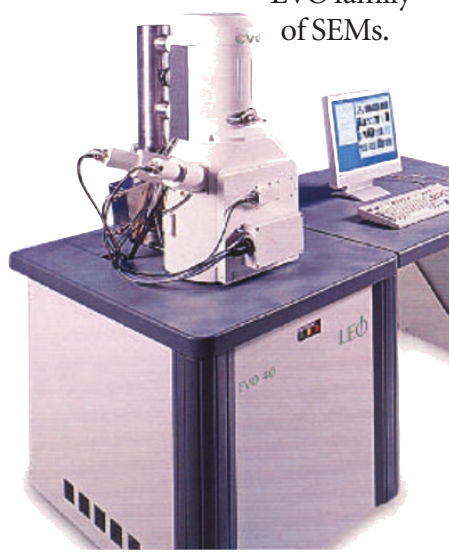

The extended pressure range enables imaging in either water vapour or air at pressures from $1 \mathrm{~Pa}$ to $750 \mathrm{~Pa}$, which allows hydrated, insulating and outgassing specimens to be imaged.LEO's VPSE detector provides true secondary electron imaging for XVP,

which they claim results in imaging under XVP mode as productive as that under high vacuum.

Any highvacuum LEO EVO microscope can be upgraded to XVP operation in the customer's laboratory.

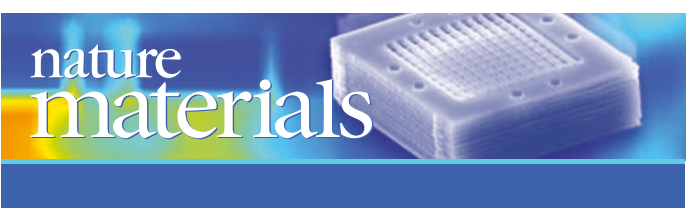

\section{New on the Market}

The 'New on the Market' section of Nature Materials is designed to give our readers access to the latest technologies, products and senvices available in the highly competitive materials science and engineering fields. With a worldwide circulation, Nature Materials is an invaluable resource for all scientists, in both academia and industry, who are active in the process of discovering and developing materials and materials-related concepts. We would like to invite you to send your Press Release Information about your new technologies, products and services for FREE inclusion in the features section 'New on the Market'.

Weblinks will accompany each PR and some pictures will also be included. As an editorial page of Nature Materials, these PRs will also appear online.

For further information please contact Gerard Preston, Senior European Advertising Manager, by email at g.preston@nature.com

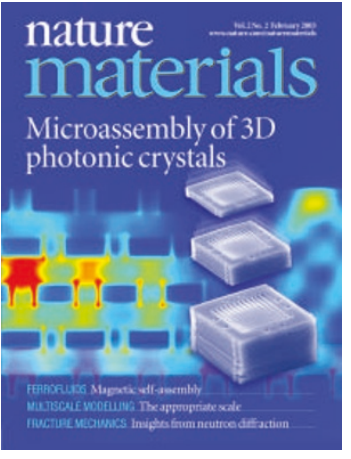

FEMLAB 3.0 COMSOL WWw.comsol.com

Mathematical modelling

\section{FEMLAB 3.0 is an}

equation-based package that uses finite-element analysis to solve models of physical phenomena to better understand a material's underlying properties. The image below shows a model of the temperature distribution and efficiency in a MEMs heat exchanger. COMSOL say their new software can generally solve problems in half the time and half the memory of its predecessor. Almost any physical phenomena can be modelled, including heat transfer, fluid flow, electromagnetics and structural mechanics. Because of the interdisciplinary nature of the software, a researcher can use the single graphical interface to couple different processes. The software will set up the relevant equations for the type

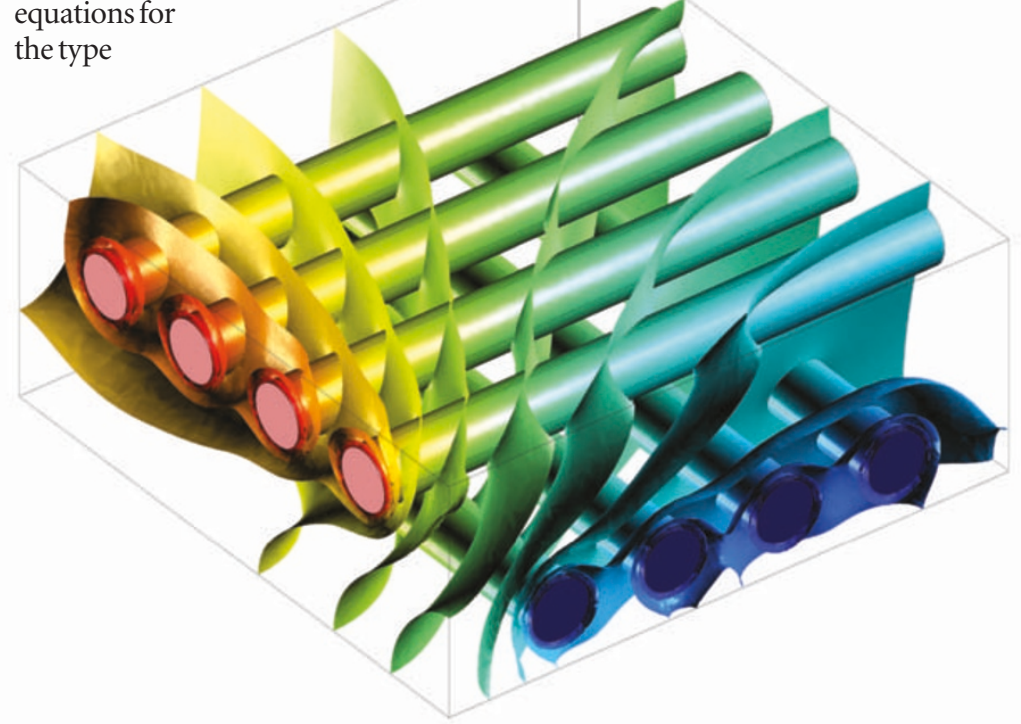

These notes are compiled in the Nature Materials office from information provided by the manufacturers. of model chosen by the user, or the user can type in their own equations directly. As many as a million degrees of freedom can be handled in areas such as structural mechanics, heat transfer and chemical convectiondiffusion, among others. Stationary, eigenvalue and time-dependent problems of this size can be handled. The solution time in these cases typically ranges from 15 minutes to two hours, depending on the solver technique and the speed of the computer. A builtin CAD editor allows twoand three-dimensional objects to be drawn, and the interface allows realtime, smooth rotation of a three-dimensional image in any direction. The program's animation capabilities

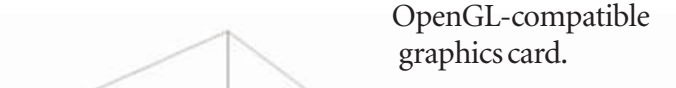

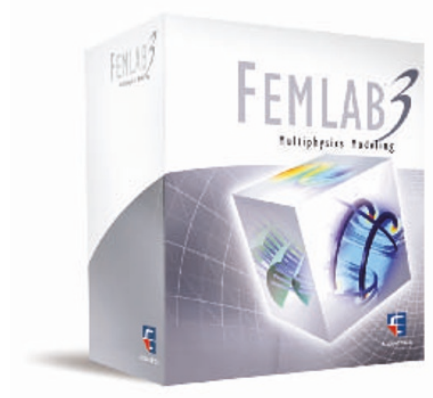

enables fast insight into a dynamic process, plus the ability to generate AVI or Quicktime movies. The new software is standalone, but can be integrated with MATLAB (from The MathWorks). System requirements are Windows 98/2000/NT 4.0/XP as well as Linux, Solaris and HP-UX. Minimum system configuration is a Pentium processor, 256M bytes of RAM ( 512 recommended) and an OpenGL-compatible graphics card.

M

